\title{
MSCT evaluation of patients with prior coronary bypass surgery: what we have and what we lack
}

\author{
Nuno Bettencourt · Amedeo Chiribiri • \\ Eike Nagel
}

Received: 1 October 2008/Accepted: 1 October 2008/Published online: 31 October 2008

(C) Springer Science+Business Media, B.V. 2008

In the study by von Kiedrowski et al. published in this issue, the clinical utility of 16-slice CT in assessing patients with prior coronary bypass surgery $(\mathrm{CABG})$ is tested.

In this retrospective single centre study, the authors describe computed tomography angiography (CTA) findings in the assessment of both the bypass grafts and the native vessels of 39 patients referred for CTA due to chest pain or inconclusive stress tests. Eighteen of those patients were furthermore referred to invasive coronary angiography (CA) after pathologic findings in CTA. This subgroup of patients with both exams performed was used to calculate MSCT accuracy for predicting significant coronary stenosis, defined by a luminal lumen narrowing $\geq 50$, using CA QCA as the gold standard. Per patient, per graft and per native vessel analyses were made.

The authors found a very good overall accuracy of CTA in the assessment of bypass grafts patency/ stenoses and an acceptable accuracy in the native vessel evaluation, with an overall very low rate of

Editorial comment to: von Kiedrowski et al. (2008) Noninvasive coronary angiography: the clinical value of multi-slice computed tomography in the assessment of patients with prior coronary bypass surgery. Int J Cardiovasc Imaging. doi: 10.1007/s10554-008-9361-x.

N. Bettencourt · A. Chiribiri · E. Nagel $(\square)$

King's College London, Strand, London, UK

e-mail: eike.nagel@kcl.ac.uk false negatives. However, $28.5 \%$ of native vessel segments were considered unevaluable.

Despite the limitations that result from the retrospective nature of the study, with obvious implications in the interpretation of the results, due to the so-called "verification bias" (that tends to overestimate sensitivity and underestimate specificity of the test), one of the advances of this study is that, differently from previous studies [1], the clinical value of CTA was assessed not only for the graft evaluation but also for the native coronary arteries assessment, namely the non-grafted native arteries and the proximal and distal segments of the bypassed vessels. For a non-invasive study in symptomatic patients after $\mathrm{CABG}$ it is important to include the assessment of native coronary arteries, as these may be responsible for the symptoms and their assessment may change treatment [2-4]. However, CT assessment of native coronary arteries in patients after $\mathrm{CABG}$ is challenging, owing to the advanced atherosclerotic disease with abundantly calcified and diffusely narrowed arteries with small dimensions [2, 3].

The diameter size, relative immobility and sparce presence of calcifications make grafts ideal for assessment by non-invasive imaging techniques. CT angiography is not limited by some of the practical disadvantages of $\mathrm{CA}$, such as the requirement for selective contrast injection. Particularly when the exact surgical history is incomplete, CT allows comprehensive graft visualization, including the site and identity of distal run-offs [3]. In this study, all 
grafts were evaluable by MSCT (in contrast to CA where seven grafts were unassessable).

Artefacts caused by metal within the vicinity of the graft prevented evaluation in $9.3 \%$ of grafts in this study. More efficient scanners, with better spatial and temporal resolution may help solving this problem by reducing hardening artefacts. In fact, recent studies based in 64-SCT confirmed that metal clips artefacts may no longer represent a major limitation for CTA using this technology [3].

The main limitation of the generalized use of CTA for this indication seems to be the clinical need for native vessel evaluation, as severe coronary calcification has a high prevalence in patients with CAD following bypass surgery. The proximal and, more importantly, the distal native vessels associated with the bypass anastomosis ("run-off vessels") are often heavily affected by calcification, precluding a correct assessment of the lumen (12\% of native run-off vessels in this study). As the authors state, this problem seems to be independent of spatial resolution, and is still a major limitation for CTA, even when more recent technology is used. (von Kiedrowski et al. this issue) [2].

Assessment of heavily calcified segments seems to be a structural problem of computed tomography and until further developments are achieved, it threats to be the major limiting factor for the broad use of this technology, in patients with severe coronary calcification, such as patients with CABG. In fact, even newer generations of MSCT scanners "do not completely detach problems dealing with hardening artefacts induced by metal clips and calcification." (von Kiedrowski et al. this issue) [3].

Radiation is another problem concerning CTA: in the present study all scans were performed using dose reduction protocols. Mean radiation exposure was estimated as $9.88 \pm 3.2 \mathrm{mSV}$. Compared to other published works using MSCT in CABG patients, this is a relatively low dose exposure. However, some of the scans excluded the proximal segment of the LIMA or RIMA even when these arterial grafts had been used for bypass surgery, explaining the differences to other studies. [2, 5]. As the authors commented, "minimization of radiation exposure, as well as optimization of the diagnostic abilities in calcified vessels remain the main goals for future MSCT technologies."
In this study unevaluable segments were considered as false positives. Thus, the results reflect the real value of this technique in a clinical setting-as patients with unevaluable segments would not be exempt from evaluation (by CA or other diagnostic technique). Using this approach, the NPV is kept very high and is one of the strong arguments for the technique. On the other hand, the high number of false positive studies, particularly in the presence of calcified disease, is an important limitation of CTA in this context, as it implies an unnecessary duplication of exams with the associated increase of radiation and contrast agent exposure.

The authors conclude that although 16-slice-CT provides "sufficient CABG assessment," the clinical value of this method is limited by its detriments in assessing the bypass anastomoses and the revascularized native vessels. The clinical indications for CTA in CABG patients are still limited, as patients with positive stress test and high pre-test probability will benefit from a direct referral for CA rather than for MSCT. However, in a selected group of patients with failed CA for bypass assessment, inconclusive stress test, inability to perform stress testing, or a generally low pre-test probability for bypass stenosis/obstruction, MSCT may have an important value for the assessment of bypass grafts. Furthermore, the ability of this method for the assessment of anatomic thoracic relations makes it of unquestionable clinical utility for the planning of reoperative cardiothoracic surgery. The decision whether to refer for CTA or CA should be based on the clinical status of the CABG patient in order to avoid duplication of radiation exposure: if an interventional approach is probable, primary CA should be the first choice.

\section{References}

1. Burgstahler C, Beck T, Kuettner A, Drosch T (2006) Noninvasive evaluation of coronary artery bypass grafts using 16-row multi-slice computed tomography with $188 \mathrm{~ms}$ temporal resolution. Int J Cardiol 106:244-249

2. Meyer TS, Martinoff S, Hadamitzky M, Will A (2007) Improved noninvasive assessment of coronary artery bypass grafts with 64-slice computed tomographic angiography in an unselected patient population. J Am Coll Cardiol 49:946950 
3. Malagutti P, Nieman K, Meijboom WB, Van Mieghem CAG, Pugliese F, Cademartiri F, Mollet NR, Boersma E, Jaegere P, Feyter PJ (2007) Use of 64-slice CT in symptomatic patients after coronary bypass surgery: evaluation of grafts and coronary arteries. Eur Heart J 28:1879-1885

4. Anders K, Baum U, Schmid M, Ropers D, Schmid A (2006) Coronary artery bypass graft (CABG) patency: assessment with high-resolution submillimeter 16-slice multidetector- row computed tomography (MDCT) versus coronary angiography. Eur J Radiol 57:336-344

5. Salm LP, Bax JJ, Jukema JW, Schuijf JD, Vliegen HW, Lamb HJ, Van Der Wall EE, Roos A (2005) Comprehensive assessment of patients after coronary artery bypass grafting by 16-detector-row computed tomography. Am Heart J 150:775-781 